\title{
PROPERTIES OF THE RECENTLY DISCOVERED ECLIPSING BINARY GSC 03421-01402
}

\author{
Christopher Lloyd ${ }^{1}$, James Screech ${ }^{2}$ \\ 1) Department of Physics and Astronomy, University of Sussex, Brighton, BN1 9QH C.Lloyd@sussex.ac.uk \\ 2) BAA Variable Star Section, Burlington House, Piccadilly, London, W1J 0DU
}

\begin{abstract}
GSC 03421-01402 is a low-amplitude eclipsing binary with a period of $7.6800475(44) \mathrm{d}$. The eclipsing binary is one component of a close pair identified in the Gaia EDR3 data with $\Delta G=0{ }^{\mathrm{m}} 45$. The light-curve suggests that the primary eclipse is total with an observed depth of 0.13 , but due to dilution the true depth could be $\sim 0.23$ or 0.37 . A weak, 0.02 , secondary eclipse is seen displaced slightly to phase 0.491 . The system is probably a detached Algol-type eclipsing binary dominated by a mid G-type primary, but until the uncertainties in the relative magnitudes and distance are resolved much remains in doubt.
\end{abstract}

\section{Introduction}

The history of GSC 03421-01402 (UCAC4 709-047369, 2MASS 08181042+5138193) is short but not lacking in complication. The star was first found to be a low-amplitude variable in December 2019 by Screech (2020) while making observations of the W UMa variable FI Lyn. The star showed an eclipse with $\Delta V=0.15$ and a full width of $\sim$ 6 hours. Extensive observations over the following year found the star mostly at a constant magnitude, but one more eclipse was recovered. An earlier observation of a possible minimum by Walter (2019) helped narrow the likely period to 7.68 and eventually led to the observation of a predicted eclipse (see Screech, 2021, for details). In addition to the three primary eclipses a possible weak secondary eclipse was also observed.

However, during this time Zhu et al. (2020) also reported the discovery of the star as an eclipsing binary, and found the same period. Zhu et al. identified the star by its Gaia DR2 name (Gaia Collaboration et al., 2018), and this is the one SIMBAD uses, but in the EDR3 catalogue (Gaia Collaboration et al., 2021) the star is identified as a close double with a separation of 0.346 arcsec. So, all ground-based measurements of this star will refer to the pair, and the relative contributions may depend on the photometric band.

Table 1: Identifiers used for GSC 03421-01402

\begin{tabular}{cccc}
\hline Name & RA & Dec & Note \\
\hline \hline GSC 03421-01402 & 081810.421 & +513819.75 & \\
UCAC4 709-047369 & 081810.4223 & +513819.492 & \\
CSTARII124.5434+51.6387 & & & Zhu et al. (2020) \\
Gaia DR2 1031338887492194944 & 081810.4079 & +513819.399 & Composite \\
Gaia EDR3 1031338887492194944 & 081810.4236 & +513819.357 & Star A \\
Gaia EDR3 1031338887493704960 & 081810.4133 & +513819.698 & Star B \\
\hline
\end{tabular}


Table 2: Observing log of the time-series runs

\begin{tabular}{ccrr}
\hline Date & JD & Hours & $\mathrm{N}$ \\
\hline \hline 2019 Dec 29 & $2458847.329-.797$ & 11.2 & 3021 \\
2020 Jan 03 & $2458852.305-.484$ & 4.3 & 1195 \\
2020 Jan 06 & $2458855.316-.680$ & 8.7 & 283 \\
2020 Jan 15 & $2458864.227-.606$ & 9.1 & 2416 \\
2020 Jan 18 & $2458867.225-.377$ & 3.7 & 304 \\
2020 Jan 19 & $2458868.235-.656$ & 10.1 & 314 \\
2020 Jan 20 & $2458869.236-.735$ & 12.0 & 1276 \\
2020 Jan 28 & $2458877.242-.791$ & 13.2 & 869 \\
2020 Feb 06 & $2458886.251-.774$ & 12.5 & 929 \\
2020 Feb 11 & $2458891.251-.775$ & 12.6 & 1230 \\
2020 Feb 20 & $2458900.297-.429$ & 3.2 & 338 \\
2020 Mar 02 & $2458911.297-.723$ & 10.2 & 973 \\
2020 Mar 06 & $2458915.289-.604$ & 7.5 & 707 \\
2020 Dec 19 & $2459203.219-.604$ & 9.2 & 948 \\
2020 Dec 20 & $2459204.216-.420$ & 4.9 & 534 \\
2020 Dec 24 & $2459208.223-.799$ & 13.8 & 1326 \\
2020 Dec 27 & $2459211.216-.380$ & 3.9 & 423 \\
2021 Jan 21 & $2459236.384-.789$ & 9.7 & 1069 \\
2021 Jan 23 & $2459238.238-.531$ & 7.0 & 770 \\
2021 Feb 03 & $2459249.308-.774$ & 11.2 & 1216 \\
2021 Feb 10 & $2459256.276-.754$ & 11.5 & 1255 \\
2021 Feb 18 & $2459264.271-.603$ & 8.0 & 874 \\
2021 Feb 22 & $2459268.320-.574$ & 6.1 & 565 \\
2021 Feb 25 & $2459271.275-.712$ & 10.5 & 1085 \\
2021 Feb 26 & $2459272.287-.710$ & 10.1 & 1050 \\
2021 Feb 27 & $2459273.278-.704$ & 10.2 & 1127 \\
2021 Mar 24 & $2459298.334-.637$ & 7.3 & 791 \\
2021 Apr 14 & $2459319.328-.578$ & 6.0 & 666 \\
2021 Apr 15 & $2459320.331-.574$ & 5.8 & 640 \\
2021 Apr 18 & $2459323.333-.565$ & 5.6 & 617 \\
\hline & & & \\
& & & \\
& &
\end{tabular}

The additional uncertainty also extends to the Gaia-based distance measurements where Bailer-Jones et al. (2021) give $553_{-80}^{+102}$ and $487_{-40}^{+77}$ pc for the brighter component (Star A) using their two methods. There is currently no Gaia parallax for the companion (Star B), but as Gaia continues its work these uncertainties will hopefully be resolved.

\section{Observations}

The photometric observations were made from a private observatory in Bedford, England using an ED70 refractor and Atik 414ex CCD from 2019 December 29 to 2020 March 6, and a ZWO ASI1600MM-C CCD from 2020 December 19, both with a $V$ filter. The exposure times were shorter for the first two runs as the main target was FI Lyn, which is 

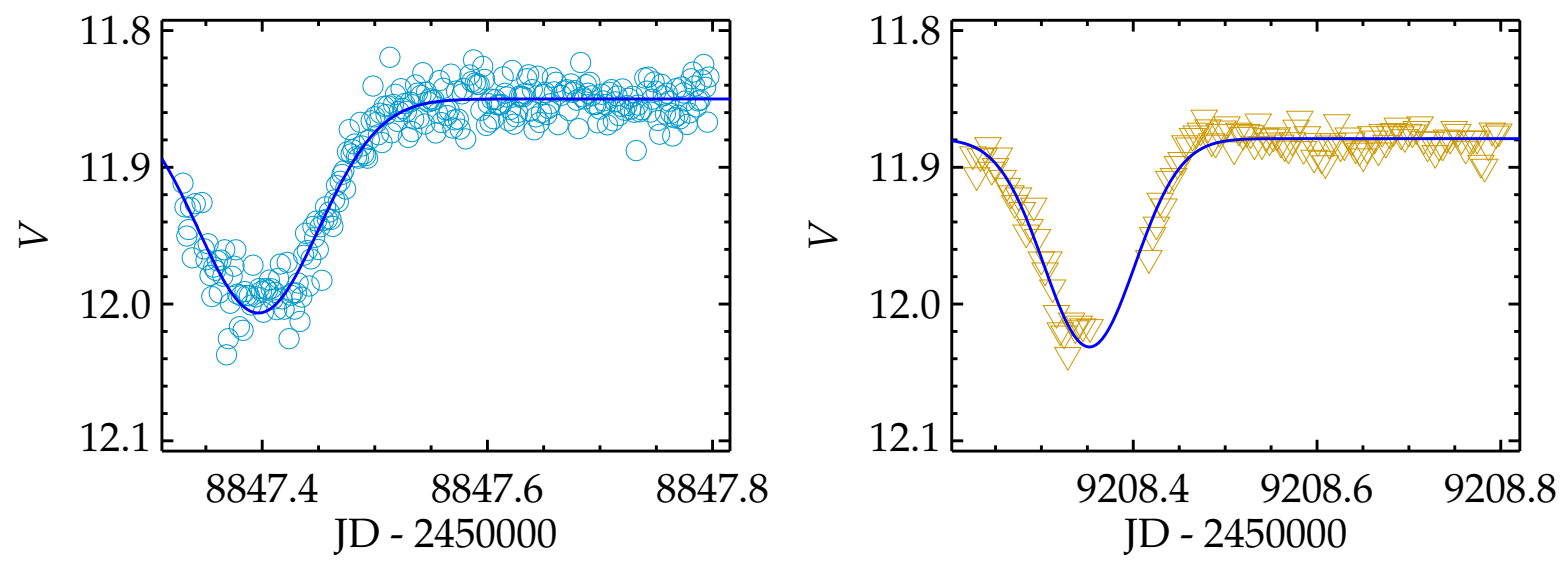

Figure 1: The first two primary eclipses observed in the time-series data with the fitted Gaussian profile.

two magnitudes brighter, and this accounts for the increased noise in the discovery light curve, shown in Figure 1. All the images were bias-, dark-subtracted and flat fielded, and then reduced using the Muniwin aperture photometry routine of the C-Munipack v2.1 software package (see also Chrastina \& Hroch, 2008), which is based on DAOPHOT (Stetson, 1987). The $V$ magnitudes were obtained by calibration with respect to HD $233508 V=9.82, B-V=0.51$, from the AAVSO comparison star sequence X24482CSA for FI Lyn. The star was observed on 30 nights between 2019 December and 2021 April, and all the observations were made in long runs, between 3 and 13 hours, which are listed in Table 2. The individual eclipses are shown in Figures 1, 2 and 3, and detail of the phase diagram around primary and secondary minimum is shown in Figure 4 using the ephemeris in Equation 1 determined later. All the data are available from the BAA VSS
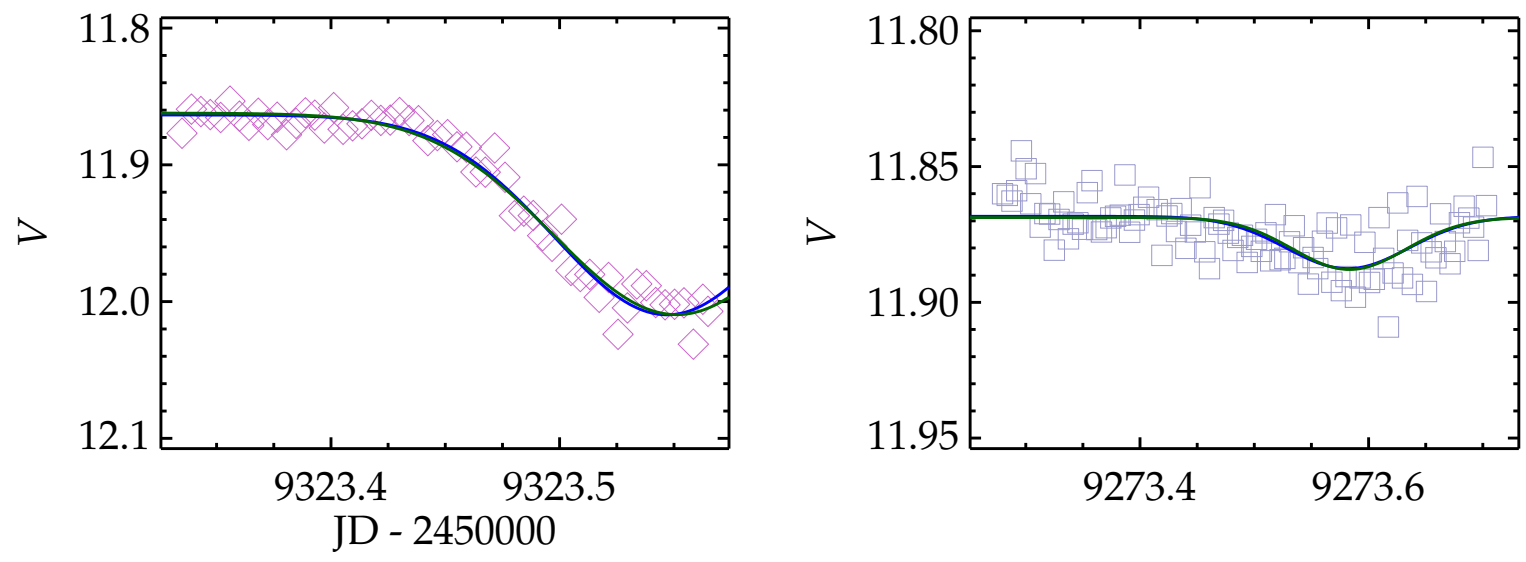

Figure 2: The third and least well observed primary Figure 3: The only time-series run that covers the eclipse in the time-series data with two slightly dif- secondary eclipse with two slightly different profiles ferent profiles with the fits using widths fixed at the with the fits using widths fixed at the values from values from the first two eclipses. the first two eclipses. 

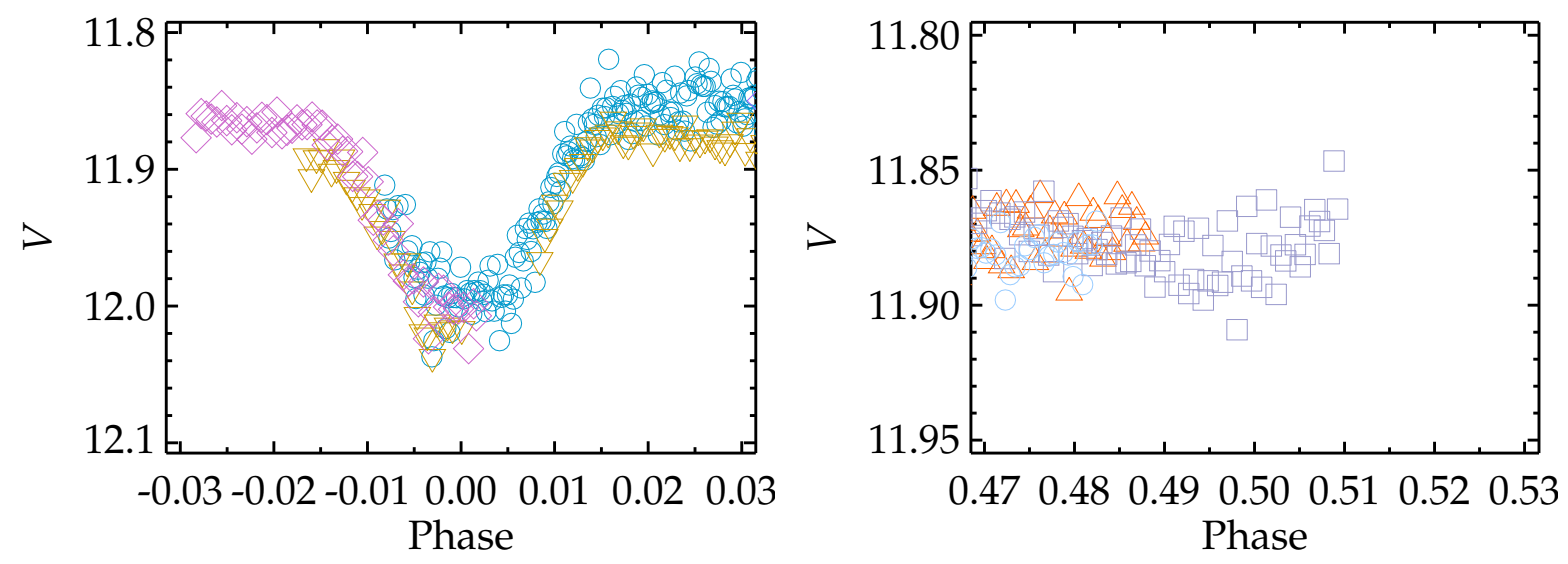

Figure 4: Detail of the phase diagram of the time-series data showing the primary eclipse (left) and secondary eclipse (right), folded on a ephemeris in Equation 1. The primary eclipse shows a distinct flattening which would indicate that it is total. The colours of the different runs are the same as in Figures 1, 2, and 3 .

Photometry Database or the AAVSO data archive.

The star has also been observed by TESS (see Ricker et al., 2015, and documentation) in Sector 20 in Full Frame Image (FFI) mode, which ran from JD 2458842.5 in two 12.4 day sections separated by a 1.5 day gap. Photometry was extracted from the FFI data using the Python Lightkurve v2 package (Lightkurve Collaboration et al., 2018) with a simple average background extraction and custom aperture. The residual orbital background variation of $\sim 0 \mathrm{~m} 01$ was removed by normalizing the light-curve using a wide median filter. The TESS light curve confirms the period and shows two primary eclipses and four secondary eclipses of 0.02 , which are slightly displaced from phase 0.5 , and support the secondary eclipse seen in the time-series data. The 30 minute cadence of the FFI data
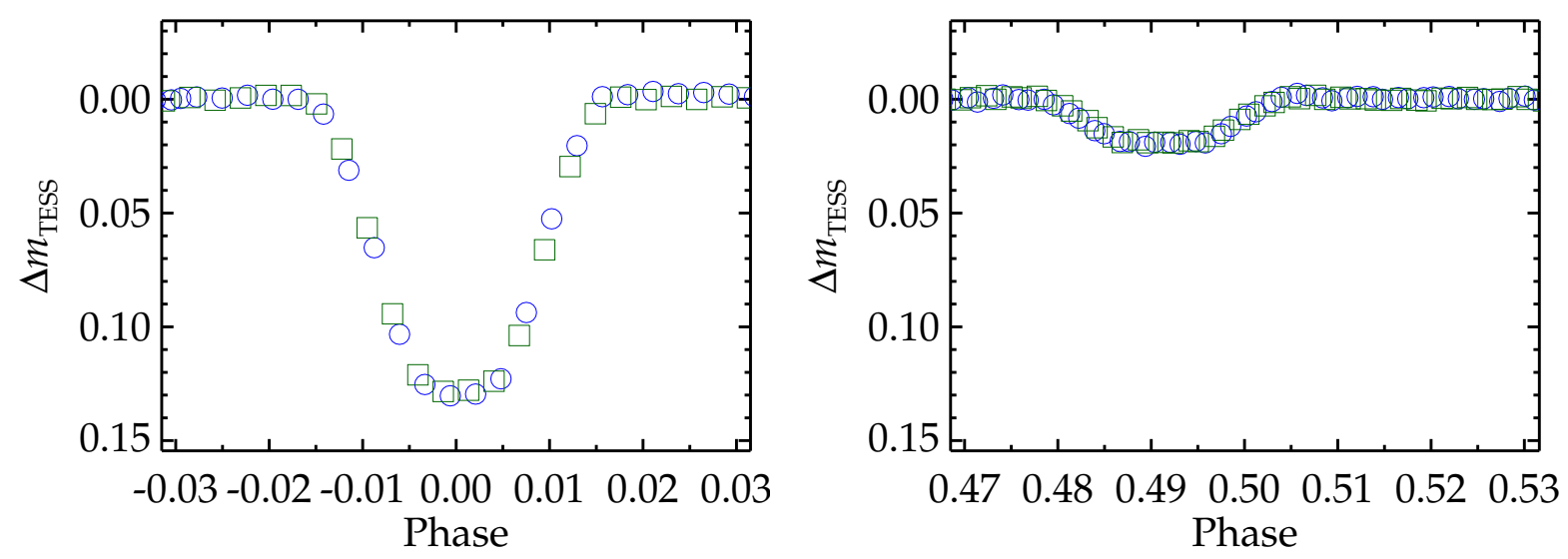

Figure 5: Detail around primary eclipse (left) and secondary eclipse (right) from the TESS data folded on the ephemeris given in Equation 1. 

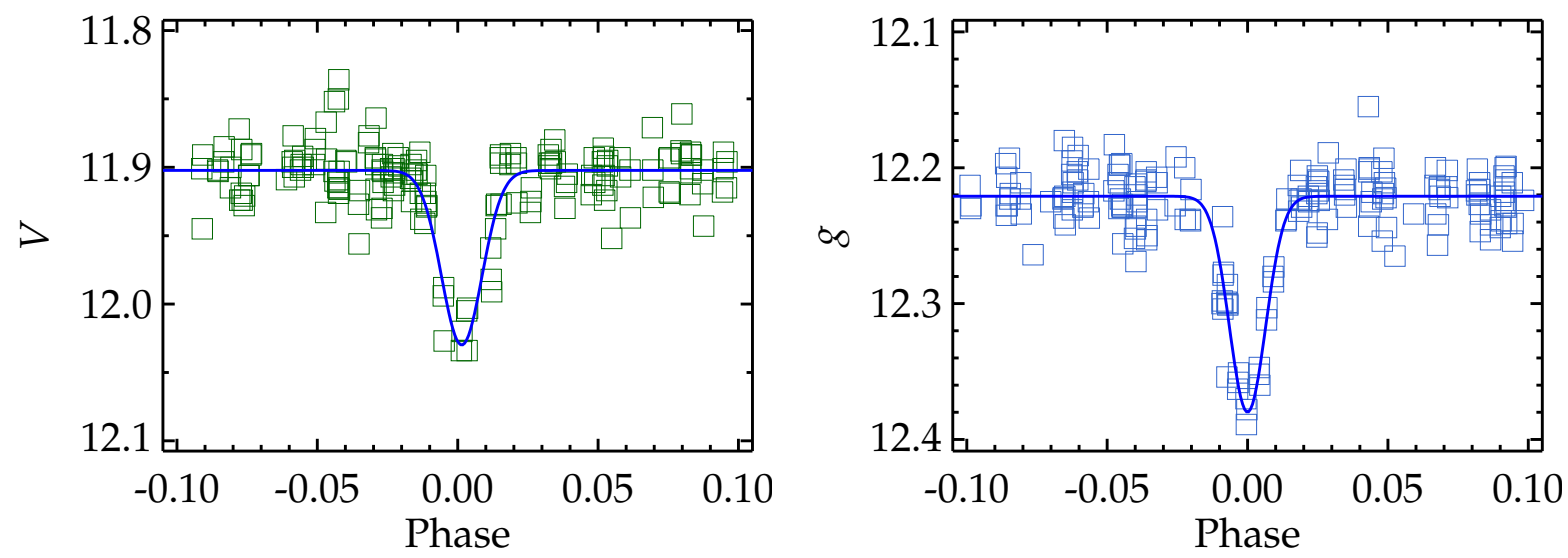

Figure 6: Phase diagram of the ASAS-SN $V$-band (left) and ASAS-SN $g$-band data (right) folded on a period of 7.6800 .

means that the minima are relative sparsely covered but nevertheless the eclipses are well defined, particularly the secondary which is exquisitely delineated. Detail of the minima is shown in the folded light-curves in Figure 5. The eclipses are clearly total.

Data have also been taken from the All-Sky Automated Survey for Supernovae (ASASSN) archives (Shappee et al., 2014; Kochanek et al., 2017), which provides up to three $V$ or $g$-band magnitudes every few days throughout the observing season. Multiple observations are typically made in the space of 0.003 or 4 minutes, although for various operational reasons not all of these survive, so on average $\sim 75 \%$ of the groups contain three points. Occasionally several groups are made in one night. Observations are available from 2015 to 2018 in the $V$-band and from 2018 to the present with the SDSS $g$ filter. The folded light-curve is shown in Figure 6.

Additional data have been taken from the Northern Sky Variability Survey (NSVS
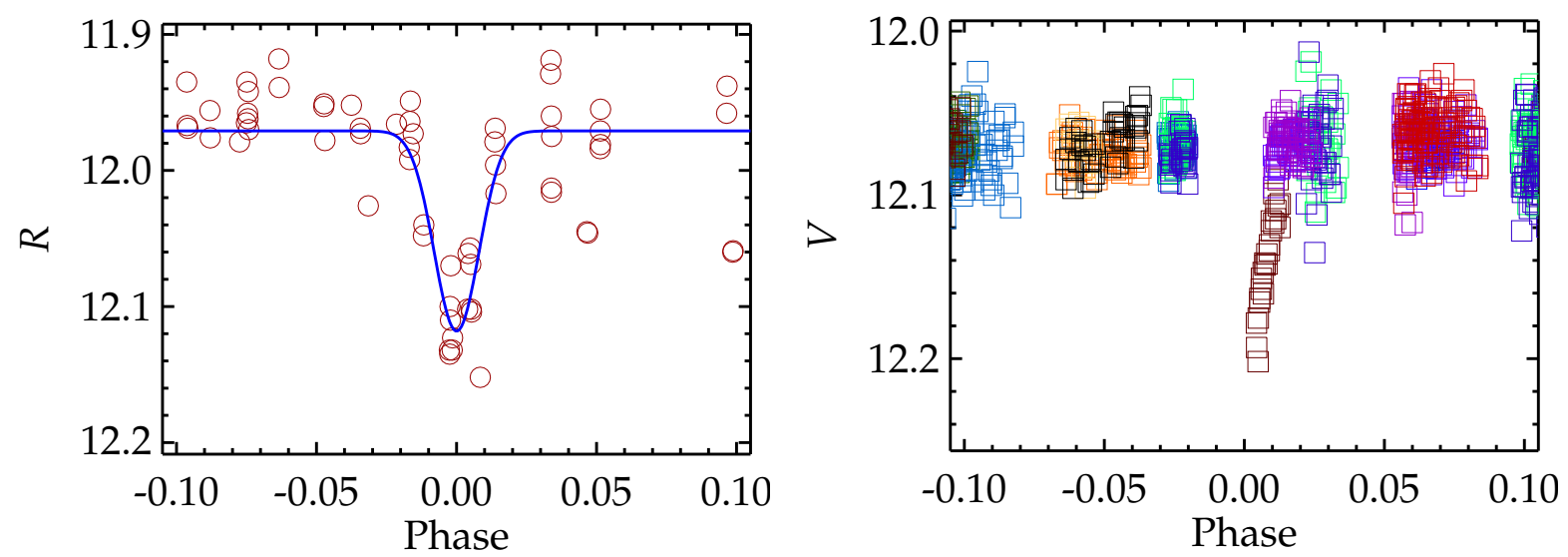

Figure 7: Detail of the phase diagram of the NSVS Figure 8: Detail of the phase diagram of the Superdata. WASP data showing the single eclipse. 
Akerlof et al., 2000; Woźniak et al., 2004) (see Figure 7) which ran during 1999-2000 and provided 850 points. Unfortunately most of the NSVS data are flagged as unreliable and/or have unacceptably large errors, so the data have been limited to those with the least damaging flags, according to the criteria of Wils et al. (2006), and with errors $<0$. 03 , leaving 317 points. One useful run has also been identified in the SuperWASP data (Pollacco et al., 2006; Butters et al., 2010) but it shows only part of primary minimum (see Figure 8).

\section{Times of minima}

For the time-series data the times of minima of the eclipses were obtained directly from the individual runs by fitting a Gaussian profile to the local out-of-eclipse level and the eclipse itself. For relatively shallow eclipses with poor signal to noise, and sparse or irregular data this is a good choice as it can use all the available data, and its natural profile is well matched to the eclipse. The data fitted are independent 11-point means of the individual observations and these have $\sigma=0.014$ for the first minimum and $\sigma=0$ m.009 for the others. None of the primary eclipses were observed completely, but the minimum was caught in all three. The last eclipse is the least well constrained so the width of the profile, which is one of the parameters fitted, was fixed at the value of the other two eclipses. These are shown in Figures 1 and 2. One of the time-series runs also covers phase 0.5 so this was examined for any sign of the secondary eclipse. There is a minimum in the data, which could be regarded as unconvincing, but it is suggestive. In the fits the width has again been fixed at the values of the two best eclipses and the timings averaged, although there is no significant difference.

For the TESS data the individual eclipses were fitted with both the Gaussian profile

Table 3: Times of minima

\begin{tabular}{ccrrrrl}
\hline HJD & Error & Min. & Cycle & \multicolumn{1}{c}{ O-C } & Band & Source \\
\hline \hline 2451489.9072 & 0.0044 & 1 & -958.0 & -0.0039 & $R$ & NSVS \\
2457411.2221 & 0.0040 & 1 & -187.0 & -0.0056 & $V$ & ASAS-SN \\
2458532.5184 & 0.0044 & 1 & -41.0 & 0.0038 & $C$ & Walter \\
2458701.4723 & 0.0025 & 1 & -19.0 & -0.0033 & $g$ & ASAS-SN \\
2458843.4863 & 0.0014 & 2 & -0.5 & -0.0702 & $C$ & TESS \\
2458847.3968 & 0.0012 & 1 & 0.0 & 0.0003 & $V$ & Screech \\
2458847.3974 & 0.0008 & 1 & 0.0 & 0.0009 & $C$ & TESS \\
2458851.1670 & 0.0020 & 2 & 0.5 & -0.0696 & $C$ & TESS \\
2458858.8485 & 0.0004 & 2 & 1.5 & -0.0681 & $C$ & TESS \\
2458862.7577 & 0.0005 & 1 & 2.0 & 0.0011 & $C$ & TESS \\
2458866.5280 & 0.0012 & 2 & 2.5 & -0.0686 & $C$ & TESS \\
2459208.3524 & 0.0015 & 1 & 47.0 & -0.0064 & $V$ & Screech \\
2459273.5827 & 0.0077 & 2 & 55.5 & -0.0565 & $V$ & Screech \\
2459323.5490 & 0.0022 & 1 & 62.0 & -0.0105 & $V$ & Screech \\
\hline
\end{tabular}

Only the primary minima are used to derive the ephemeris. 
and using the Kwee - van Woerden (Kwee \& van Woerden, 1956), which although more general was very close to the lower limit of the number of points that could be realistically used. In practice both produced almost identical results and were well within the errors, but the Kwee - van Woerden values are preferred. The folded light-curves are shown in Figure 5. From the Gaussian fits to the time-series data the depth of the primary minimum is $0.15 \pm 0.01$ in $V$, but as the eclipse is total this may be a slight overestimate. The TESS data give a depth of 0.13 , and although this is a panchromatic red magnitude it is better determined than the depth from the time-series data, and probably not too dissimilar to $\Delta V$. The depth of the secondary eclipse is 0.02 from TESS. The full width of the primary eclipse is 6.0 hours and the FWHM is 3.1 hours from both the TESS and time-series data, but the secondary minimum is clearly narrower with a full width near 4.8 hours, presumably due to the eccentricity of the orbit. A more detailed treatment of the TESS data and an a photometric solution will be published elsewhere.

With a preliminary ephemeris it was possible to fold the NSVS and ASAS-SN data, and attempt to extract composite eclipse timings from these data. The profile fitting was made with a range of periods close to 7.68 to test the sensitivity to the period and establish the stability of the derived times of minima. The ASAS-SN data shows some sensitivity to the period chosen as the $V$ and $g$ data were taken over four and three years respectively, even so this variation has little impact on the derived times of minima or the uncertainty. These minima are shown in Figure 6. For the NSVS data, the quality of the fit and the reliability of the minimum is insensitive to the period as the data were taken in $\sim 350$ days. The NSVS minimum is shown in Figure 7 . For consistency all the composite timings were derived from data folded on 7 d.6800. A further timing has been measured from Walter (2019)'s light-curve using the same Gaussian fitting method as before, and the timings are collected in Table 3. Another eclipse has been identified in the SuperWASP data (Pollacco et al., 2006; Butters et al., 2010) as shown in Figure 8, but this is incomplete and does not cover the minimum.

\section{The O-C diagram}

The linear ephemeris was determined from a weighted fit to the primary minima as the secondary is clearly displaced from phase 0.5 . The ephemeris of primary minimum is

$$
H J D_{\mathrm{MinI}}=2458847.3965(4)+7.6800475(44)
$$

and secondary minimum is

$$
H J D_{\mathrm{MinI}}=2458851.1682(6)+7.6800475(44)
$$

with the period fixed, making the phase of secondary eclipse 0.491 . The detail around the primary and secondary minima of the time-series data folded on this ephemeris is shown in Figure 4 and similarly for the TESS data in Figure 5, where the displacement of the secondary eclipse is obvious. The $\mathrm{O}-\mathrm{C}$ diagram constructed using this ephemeris is shown in Figure 9. 


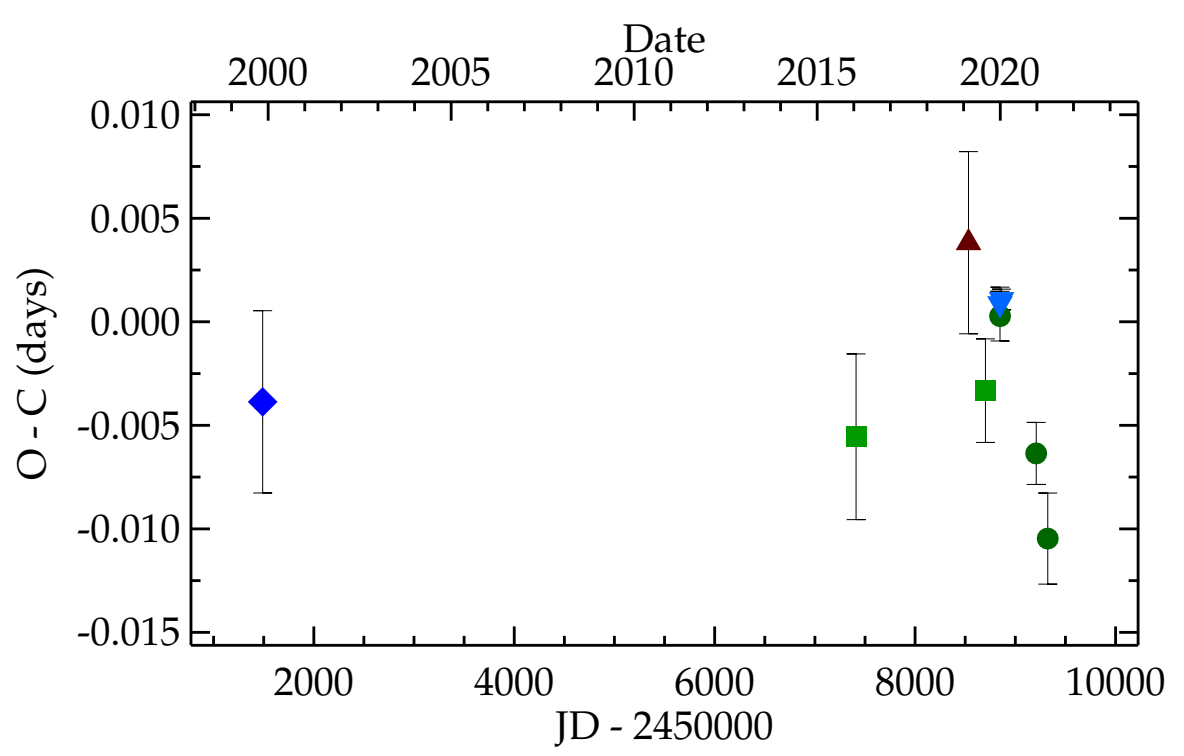

Figure 9: O-C diagram of the times of minima from Table 3 showing the NSVS point (diamond), ASASSN $V$ and $g$-band data (squares), Walter minimum (up-triangle), TESS data (down-triangles) and the times-series data (circles). The earliest point from the SuperWASP primary eclipse and all the secondary minimum lie outside the plot.

\section{Discussion}

Essentially all ground-based measurements of the star will be a blend of the two components identified in the Gaia EDR3 data. The only direct observation of the magnitude difference between the two stars is in the EDR3 data where the $G$ magnitudes are 12.209(4) and 12.656(7), giving $\Delta G=0.45$, but of course it is not clear which is the eclipsing binary. Perversely, the $B P$ and $R P$ magnitudes of the two components are identical within the errors at $B P=12.105(4)$ and $R P=11.195(7)$ respectively, which may indicate confusion, or a composite spectrum. Assuming that the difference is 0.45 , and that the eclipse depth in $G$ is the same as seen by TESS at $0 .{ }^{\mathrm{m}} 13$, then it is possible to calculate the undiluted eclipse depths for both stars. As the relative flux contributions of the two stars are 0.60 and 0.40 then the undiluted eclipses will be approximately slightly less and slightly more than twice $0 .{ }^{\mathrm{m}} 13$, for the brighter and fainter star respectively. The combined magnitude is $G=11.66$ so the eclipsed magnitude is $G=11.79$. For Star A the uneclipsed magnitude is $G=12.21$ and the eclipsed can be calculated as $G=12.44$, making the real eclipse depth 0.23 . The same exercise with Star B leads to an undiluted eclipse depth of $0 .{ }^{\mathrm{m}} 37$. Both possibilities are realistic so either star could be the eclipsing binary. The undiluted depth of the secondary eclipse remains small at 0.03 and 0.05 in both cases.

If the magnitude difference of the close pair, $\Delta G=0.45$, can be directly related to the difference in $V$ then the combined $V=11.90 \pm 0.025$ from ASAS-SN for the close pair converts to $V=12.45$ and $V=12.90$ for Star A and B respectively. The extinction is relatively low with $E_{\mathrm{g}-\mathrm{r}}=0.06 \pm 0.02$ (Green et al., 2019) so with $E_{\mathrm{B}-\mathrm{V}} \approx E_{\mathrm{g}-\mathrm{r}}$ at small values and $R_{\mathrm{V}}=3.1$, and using the uncharacteristically poor distance of $500 \pm 100 \mathrm{pc}$ 


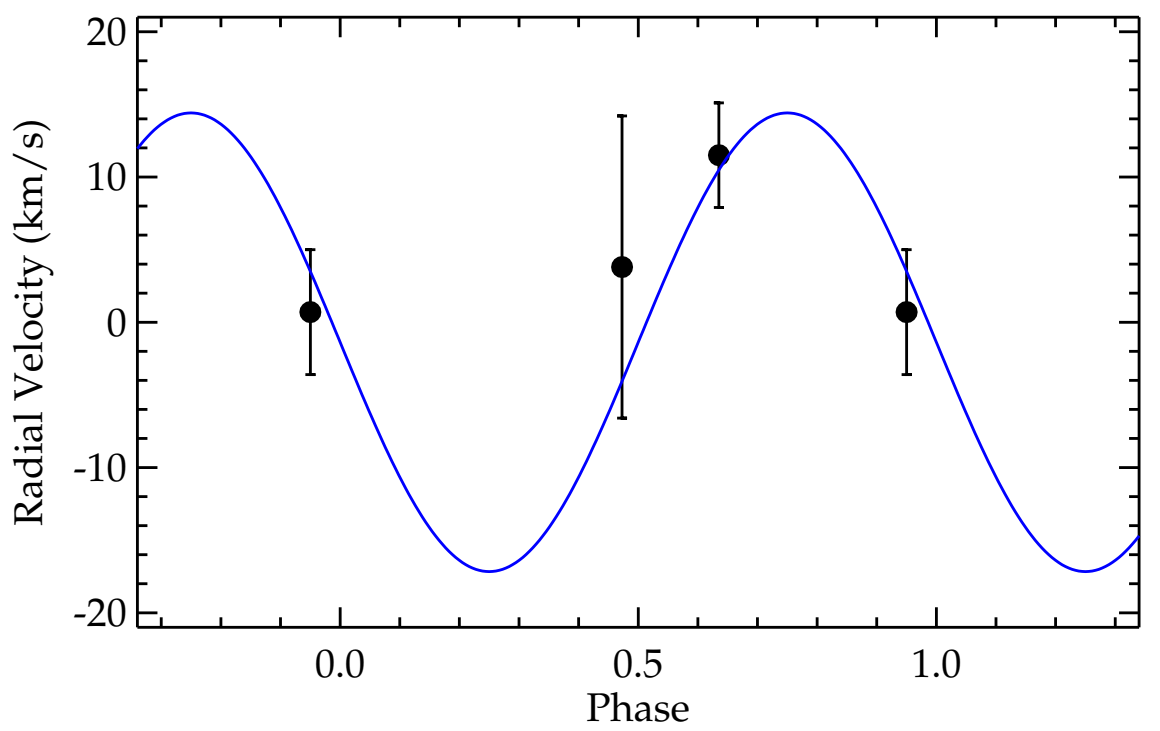

Figure 10: The velocities phased on the photometric solution in Equation 1. The line is a marginally better fit than a constant velocity but it suggests than any variation is low.

from Bailer-Jones et al. (2021), gives $M_{\mathrm{V}}=3.75$. Using to the Rochester calibration this absolute magnitude corresponds to an F6 main sequence star with $T_{\text {eff }}=6340 \mathrm{~K}$ (see Pecaut \& Mamajek, 2013, for details). The EDR3 temperature calibration gives $T_{\text {eff }}=$ $5500 \mathrm{~K}$ for Star A and for the combined light DR2 gives $T_{\text {eff }}=5377 \mathrm{~K}$ (Gaia Collaboration et al., 2018), and various calibrations of the LAMOST spectra give $T_{\text {eff }} \approx 5650 \mathrm{~K}$ (e.g., Xiang et al., 2019). These temperatures correspond more closely to a G6V star, and a spectral type of G7 is given by Bai et al. (2018) based on LAMOST spectra. So, Star A, and the combined light, which is dominated to some extent by Star A, suggest that the star has a spectral type near G6, for which the Rochester calibration gives $M_{\mathrm{V}}=5.13$. The difference between this and the photometric value is 1.4 , which most likely means that Star A has multiple components. There is currently no distance for Star B so it is impossible to make a similar comparison, but if the two are physically linked (the minimum separation would be $\sim 200 \mathrm{AU}$ ) then it is also about one magnitude brighter that a single G6V star. Obviously all this depends on the correct relative magnitudes and distance.

Three radial velocities are available for GSC 03421-01402 from the LAMOST DR5 catalogue Luo et al. (2019), which almost constitute an orbit. However, these velocities are the composite of three stars, Star A and Star B, either of which could be the eclipsing binary. They are shown in Figure 10 phased on the photometric solution together with the best fit circular orbit, which has $K=16 \pm 10 \mathrm{~km} \mathrm{~s}^{-1}$ and $\gamma=-1 \pm 6$. The fit is hardly convincing but it has $\chi_{\nu}^{2}=1.1$ compared with $\chi_{\nu}^{2}=1.9$ for a constant velocity with $\gamma=7 \pm 3$, so some variation may have been detected. Given the uncertainty over blending and resolution they add little at this stage but do suggest that any variation is likely to be low.

Irrespective of the confusion around blending, distance or which star is the binary, 
the light curve does provide some information from eclipse being total. The first and second contact points occur at $\phi \approx 0.015$ and 0.005 respectively, which immediately gives $r_{1} / r_{2} \sim 2$ for $i=90^{\circ}$. If the system contains G-type stars as suggested earlier or is dominated by a $\mathrm{G}$ star then the total mass is likely to be $1-2 M_{\odot}$, which suggests that $a \sim 20 R_{\odot}$, giving $r_{1}+r_{2} \sim 2 R_{\odot}$. The general appearance of the light curve suggests that the system is detached, and it also seems unlikely that an eccentric binary would be evolved, so the system probably contains two main-sequence stars. The primary eclipse would then be a transit and the primary component would be the hotter and larger star, but the luminosity ratio will depend on the third light contribution.

\section{Conclusion}

New time-series and TESS observations of GSC 03421-01402 show it is a low amplitude eclipsing binary and with additional photometry from ASAS-SN and NSVS a period of 7.6800475(44) has been determined. The eclipsing binary is one of the components of a close pair identified in the Gaia EDR3 data and the diluting effect of the companion has created some uncertainty in the Gaia EDR3 photometry, and in the parallax and associated distance measurement. The light-curve suggests that the primary eclipse is total (see Figures 4 and 5) and a weak, $\Delta m=0$. 02 , secondary eclipse is seen displaced to phase 0.491 . The observed eclipse depth is 0.13 but due to dilution the true depth could be $\sim 0.23$ or 0.37 depending on whether the binary is Star A or B. The system is probably a detached Algol-type eclipsing binary (EA/DM) with two main-sequence components. On balance the eclipsing binary is probably the brighter component of the pair (Star A) as the calculated absolute magnitude is 1.4 brighter than a main-sequence star of the observed spectral type, G6, although very little in known about Star B. No firm conclusions are possible until the uncertainties in the relative magnitudes and distance are resolved.

Acknowledgements: The authors appreciate helpful comments from the referee. The authors are pleased to acknowledge use of NASA's Astrophysics Data System Bibliographic Services. This research has made use of the SIMBAD database and the VizieR catalogue access tool, CDS, Strasbourg, France (DOI: $10.26093 /$ cds/vizier). The authors are pleased to acknowledge access to the WASP DR1 data as provided by the computing and storage facilities at the CERIT Scientific Cloud, operated by Masaryk University, Czech Republic. This paper includes data collected by the TESS mission, which are publicly available from the Mikulski Archive for Space Telescopes (MAST). Funding for the TESS mission is provided by NASA's Science Mission directorate.

\section{References}

Akerlof, C., Amrose, S., Balsano, R., et al., 2000, AJ, 119, 1901

Bai, Y., Liu, J., Wicker, J., et al., 2018, ApJ Suppl, 235, 16

Bailer-Jones, C. A. L., Rybizki, J., Fouesneau, M., et al., 2021, AJ, 161, 147 
Butters, O. W., West, R. G., Anderson, D. R., et al., 2010, AधAA, 520, L10

Chrastina, M. \& Hroch, F., 2008, Open European Journal on Variable Stars, 95, 21

Gaia Collaboration, Brown, A. G. A., Vallenari, A., et al., 2018, A\&A, 616, A1

Gaia Collaboration, Brown, A. G. A., Vallenari, A., et al., 2021, A\&A, 649, A1

Green, G. M., Schlafly, E., Zucker, C., et al., 2019, ApJ, 887, 93

Kochanek, C. S., Shappee, B. J., Stanek, K. Z., et al., 2017, PASP, 129, 104502

Kwee, K. K. \& van Woerden, H., 1956, BAN, 12, 327

Lightkurve Collaboration, Cardoso, J. V. d. M., Hedges, C., et al., 2018, Lightkurve: Kepler and TESS time series analysis in Python

Luo, A. L., Zhao, Y. H., Zhao, G., et al., 2019, VizieR Online Data Catalog, V/164

Pecaut, M. J. \& Mamajek, E. E., 2013, ApJ Suppl, 208, 9

Pollacco, D. L., Skillen, I., Collier Cameron, A., et al., 2006, PASP, 118, 1407

Ricker, G. R., Winn, J. N., Vanderspek, R., et al., 2015, Journal of Astronomical Telescopes, Instruments, and Systems, 1, 014003

Screech, J., 2020, VSS Circ, 183, 43

Screech, J., 2021, VSS Circ, 188, 37

Shappee, B. J., Prieto, J. L., Grupe, D., et al., 2014, ApJ, 788, 48

Stetson, P. B., 1987, PASP, 99, 191

Walter, F., 2019, Czech Astronomical Society VSES Archive - GSC 03421-01402 Lyn

Wils, P., Lloyd, C., \& Bernhard, K., 2006, MNRAS, 368, 1757

Woźniak, P. R., Vestrand, W. T., Akerlof, C. W., et al., 2004, AJ, 127, 2436

Xiang, M., Ting, Y.-S., Rix, H.-W., et al., 2019, ApJ Suppl, 245, 34

Zhu, J., Zhang, H., Liang, E.-S., et al., 2020, AJ, 159, 172 